\section{T cells in the gut promote CVD and slow metabolism}

The role of immune cells, such as T cells and macrophages, in adipose tissue in whole-body metabolism is well known. However, whether immune cells in the gut are involved in regulating systemic metabolism, in addition to their function as a first line of defence against gut bacteria, is less clear. Swirski and colleagues now show that a subset of immune cells known as integrin $\beta 7^{+}$natural gut intraepithelial T lymphocytes (natural IELs), which are present throughout the small intestine, modulate systemic metabolism and contribute to cardiovascular disease (CVD) by limiting the bioavailability of the incretin hormone glucagon-like peptide 1 (GLP1).

The researchers found that, in addition to directing immune cells to the gut, integrin $\beta 7$ regulates systemic metabolism. Mice deficient in integrin $\beta 7\left(\operatorname{tg} b 7^{---}\right)$had heightened metabolism, with more energy expenditure and heat production than wild-type mice. Furthermore, when $\lg g 7^{-/-}$ mice were fed a high-fat and high-sugar diet, they were resistant to obesity, hypercholesterolaemia, diabetes mellitus, hypertension and atherosclerosis. Treatment of $\mathrm{Ldlr}^{-1-}$ mice with anti-integrin $\beta 7$ antibodies also led to improved glucose tolerance and attenuated atherosclerosis.

Next, Swirski and colleagues demonstrated that the effects of integrin $\beta 7$ deficiency were mediated by the reduced recruitment of natural IELs to the gut and that the protection from cardiometabolic disease in the absence of natural IELs was mediated by increased systemic levels of GLP1, a hormone produced by enteroendocrine $L$ cells and which has important beneficial effects on metabolism. Natural IELs express high levels of the GLP1 receptor (GLP1R), and the research team found that GLP1R deficiency in these cells led to increased plasma levels of GLP1. In addition, Itgb7 $7^{-/}$mice with GLP1R deficiency in leukocytes had improved glucose tolerance, less hypercholesterolaemia and developed smaller atherosclerotic lesions with fewer infiltrated leukocytes than control ltg $^{-1-}$ mice. These findings indicate that natural IELs act as regulators to slow systemic metabolism by modulating enteroendocrine activity and limiting GLP1 bioavailability.

On the potential clinical translation of their findings, Swirski says that the first question to answer is whether any of the links between natural IELs and GLP1 bioavailability exist in humans and, if confirmed, to test whether blocking the recruitment or function of natural IELs can be a viable treatment for CVD. "GLP1R agonists, such as liraglutide, are already available for the treatment of diabetes," says Swirski. Antibodies blocking integrin $\beta 7$ are also clinically available, such as vedolizumab for the treatment of ulcerative colitis and Crohn's disease. "However, vedolizumab may not work for CVD because it targets a $\beta 7$ heterodimer that is not expected to block natural IEL recruitment," points out Swirski. Another anti-integrin $\beta 7$ antibody, etrolizumab, is being tested in a phase III trial for ulcerative colitis and Crohn's disease.

Irene Fernández-Ruiz

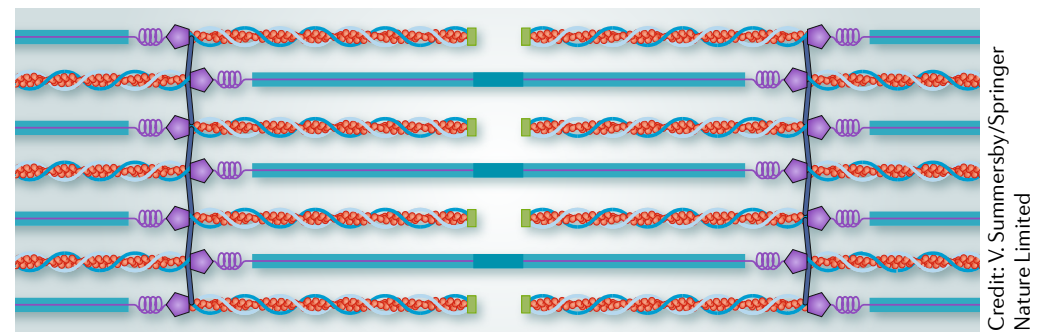

CARDIOMYOPATHIES

\title{
Modulating myosin function to treat hypertrophic cardiomyopathy
}

Hypertrophic cardiomyopathy (HCM) is typically caused by gene mutations that alter proteins in the sarcomere, the contractile apparatus of the heart, with loss-of-function mutations in MYBPC3 (which encodes cardiac myosin binding protein $\mathrm{C}$; $\mathrm{cMyBPC}$ ) and missense mutations in $\mathrm{MYH7}$ (which encodes myosin heavy chain 7) accounting for $>85 \%$ of genetic causes of this condition. However, the precise mechanisms by which these mutations lead to the cardiac hypercontractility and poor relaxation that are characteristic of HCM are unclear. A new study published in Science Translational Medicine now shows that MYBPC3 mutations cause increased contractility, reduced relaxation and excessive energy consumption of cardiac muscle by disrupting normal myosin conformations. "Our findings define a fundamental mechanism by which hearts relax and demonstrate that HCM-associated mutations in $M Y B P C 3$ cause disease by limiting normal relaxation," remarks Christopher Toepfer, first author on the paper.

Myosin is the molecular motor of the sarcomere, whereas cMyBPC is thought to act as the molecular brake. Using mouse models and human muscle fibres engineered to contain MYBPC3 mutations associated with HCM in humans, as well as a biochemical assay to assess myosin activity and ATP consumption, Toepfer and colleagues found that mutations in $M Y B P C 3$ reduced the amount of $\mathrm{CMyBPC}$ in the sarcomeres, which resulted in increased myosin contractility. The severity of cardiomyocyte phenotypes was dependent on the quantity of cMyBPC in the sarcomeres. Genetic repression of myosin motor function in mice that were engineered to carry a human $M Y H 7$ mutation improved the hypercontractile phenotype caused by cMyBPC deficiency, suggesting myosin dysregulation as the underlying mechanism in the pathogenicity of cMyBPC depletion. Furthermore, MYBPC3 mutations also impaired myosin functions during relaxation by destabilizing the myosin super-relaxed conformation - a state of dual inactivation of myosins with both ATPases inhibited, which is associated with energy conservation - and promoting a more active state that enables ATP hydrolysis and thin filament interactions.

Finally, Toepfer and colleagues demonstrated that a single pharmacological treatment targeting myosin function can correct the sarcomere dysfunction caused by MYBPC 3 mutations. The myosin ATPase inhibitor MYK-461, which is in clinical trials for the treatment of HCM, normalized the contraction and relaxation, as well as the alterations in myosin conformation, in mouse and human cardiomyocytes with MYBPC3 mutations. "The study shows a promising approach to fix the basic abnormality in HCM: target the molecular motor with drugs that reduce excessive contraction and improve relaxation," highlights Toepfer. "This potential treatment would enable the first precision medicine in HCM that directly addresses the molecular mechanism of disease, not just symptoms," he adds.

Irene Fernández-Ruiz

ORIGINAL ARTICLE Toepfer, C. N. et al. Hypertrophic cardiomyopathy mutations in MYBPC3 dysregulate myosin. Sci. Transl Med.11, eaat1199 (2019) 